\title{
Determination of Tensile and Compressive Moduli of Polymer Composites Using Three Points Cantilever
}

\author{
Shirin Rahmatpanah ${ }^{1}$, Mohmad Ghane ${ }^{2}$, Alireza Rahmatpanah ${ }^{3}$ \\ ${ }^{1}$ Science in Textile Engineering, Isfahan University of Technology, Isfahan, Iran \\ ${ }^{2}$ Textile Engineering, University of Leeds, Leeds, England \\ ${ }^{3}$ Civil Engineering, University of Qom, Qom, Iran
}

\section{Email address:}

sh.rahmatpanah@gmail.com (S. Rahmatpanah),m-ghane@cc.iut.ac.ir (M. Ghane),rahmatpanah@ut.ac.ir (A. Rahmatpanah)

\section{To cite this article:}

Shirin Rahmatpanah, Mohmad Ghane, Alireza Rahmatpanah. Determination of Tensile and Compressive Moduli of Polymer Composites Using Three Points Cantilever. American Journal of Mechanical and Industrial Engineering. Vol. 4, No. 1, 2019, pp. 21-27. doi: 10.11648/j.ajmie.20190401.13

Received: January 2, 2019; Accepted: March 6, 2019; Published: May 23, 2019

\begin{abstract}
In this work a technique is proposed to use bending test in order to determine the tensile and the compressive moduli of plastic sheets as well as polymer composites. A two- component free ends beam deflected in a three point cantilever is used to determine tensile and compressive moduli. Using small deflection equations and the theory of two- component beam, tensile and compressive moduli are then determined via computer program. The beam is composed of two components; spring steel and polymeric strip of the same width. These two components are bonded together with special glue. Two different types of material sheets were tested. The results show that both materials have different tensile and compressive modulus. To verify the model, uniaxial tensile tests were also performed. Taking the values of uniaxial tensile tests as reference showed acceptable accuracy at the proposed method.
\end{abstract}

Keywords: Tensile Modulus, Compressive Modulus, Flexural Test, Small Deflection, Two Components Beam, Polymer Composites

\section{Introduction}

Young's modulus E(n) for an elastic material is ratio of the uniaxial stress applied along the direction of a unit vector $n$ to the longitudinal strain in the direction $\mathrm{n}$ [1]. So tensile and compressive moduli of materials are important values which are related to the deformation of materials under tensile and compressive load in the defined direction. Stiffness of a material is measured by bending rigidity which is the product of moment of inertia and elastic modulus. In isotropic materials it is assumed that tensile and compressive moduli are identical. However, most of the materials such as plastic sheets, reinforced composites etc. are anisotropic in nature. These kinds of materials may behave differently in tensile stress and compressive stress. Thus it is necessary to study and investigate the mechanical behavior of materials in different load directions [2-3]. Tensile modulus can be measured relatively easily, however, because of some difficulties such as buckling; it is extremely difficult to measure the direct axial compressive stress.

The principles of cantilever have been used widely to study the flexural behavior of the composite materials or to measure the bending modulus and young modulus of such materials. In the bending test, both tension and compression are involved. In most of the bending experiments, it is assumed that the tensile and the compressive moduli of the materials are the same. However in anisotropic materials differences between these two moduli may be observed [4-7].

The main aim of this paper is to report a novel technique to determine the tensile and the compressive modules of plastic sheets including reinforced composites as anisotropic homogeneous materials. To do this, a two components beam is deflected in a bending test and the load-deflection relation is recorded. Using the classical theories of elasticity and equivalent section, tensile and compressive modules are then calculated.

In the same previous work [8] a simple cantilever was used 
to calculate tensile and compressive moduli of the plastic materials. The cantilever was fixed at one end in the jaws of a vice and a vertical load was applied on the free end of the cantilever. However some errors were involved in the experiment. The concentration of stress at the fixed end at the jaws of the vice was one of the sources of error. It was also difficult to apply the vertical load at the free end of the cantilever precisely due to slippage at the contact point.

To prevent these errors, in this work, three points cantilever is proposed. A tensile tester is also adopted to perform flexural test. The beam is simply supported at the ends and deflected at the middle by a vertical load.

\section{Theory}

\subsection{General Theory}

In this experiment three points cantilever beam was used. The beam is simply supported at the ends and a concentrated downward force, $\mathrm{P}$, is applied at the middle of the beam. In classical theories of deflection of the cantilevers it can be shown that the curvature of the neutral axis is given by equation 1 [9-10].

$$
\frac{1}{\rho}=\frac{M(x)}{E I}
$$

Where $M$ is the moment, $\rho$ is the radius of curvature, $E$ is the elastic modulus and $\mathrm{I}$ is the moment of inertia of the cross section of the beam. In analytic geometry it is shown that, in Cartesian coordinates, the curvature of a line is defined by equation 2 .

$$
\frac{1}{\rho}=\frac{\frac{d^{2} y}{d x^{2}}}{\left[1+\left(\frac{d y}{d x}\right)^{2}\right]^{3 / 2}}
$$

Where $\mathrm{x}$ and $\mathrm{y}$ are the coordinates of a point on a bent curve. If the length of the beam is very large, the slop of tangent to the curve at any point $(\mathrm{dy} / \mathrm{dx})$ is very small. In this case the value of the square of $(\mathrm{dy} / \mathrm{dx})$ can be neglected, in equation 2 , and the deflection is carried to a small deflection in the form of equation 3 ;

$$
\frac{d^{2} y}{d x^{2}}=\frac{M(x)}{E I}
$$

Appling the classical elastic theory to a two simply supported beam deflected at the middle by a vertical load, $\mathrm{P}$, the value of maximum deflection at the middle of the beam, $\mathrm{D}$, can be calculated as;

$$
D=\frac{P L^{3}}{48 E I}
$$

Where, $\mathrm{L}$ is the length of the beam.

\subsection{Two- Component Beam Theory}

Consider a two- component beam with the rectangular cross section and identical width of (b), (Figure 1). The beam is composed of two components; a spring steel strip and a composite (reinforced plastic) strip of the same width. These two components are bonded together with special glue so that no slippage occurs between them during the bending test. When a material is subjected to bending, one side of the section withstands tension while the other side away from the neutral axis is in compression. Therefore, during bending, if the neutral axis is set to intersect the steel section, the other section of the beam undergoes pure compression or pure tension depending on whether it is above or below the neutral axis. Material No. 1 is spring steel and the material No. 2 is reinforced plastic. The thickness of spring steel and composite are shown by (a) and (c) respectively.

Certain assumptions have been made such as;

1. The spring steel is an isotropic material thus; the tensile and the compressive moduli are identical;

2. The cross section of the beam remains planar during bending hence theory of solid beam can be applied;

3. Two materials have linear behavior in elastic region;

4. The weight of the beam is neglected.

The thickness of spring steel, a, is chosen in such a value so that the neutral axis intersect the spring steel region. In this way, during the bending test, the composite part is situated on one side of the neutral axis and will be under pure compression or pure tension, depending on its geometrical position. For simplification of the procedure, we used transformed section. The transformation of the section is accomplished by changing dimension of the cross section parallel to the neutral axis proportional to the ratio of elastic moduli of two materials. Figure 2 shows a T-shape equivalent transformed section of the spring steel only. The equivalent width, $\mathrm{d}$, of the composite can be calculated using Equation (5):

$$
d=b \times \frac{E}{E_{s}}
$$

Where $E_{\mathrm{s}}$ and $\mathrm{E}$ are the elastic young's moduli of the spring steel and the composite respectively. In this way the problem of the bending of a two components beam is carried to the problem of the bending of a T-section beam of the spring steel only.

In the beam with the transformed section, strains and stresses vary linearly from its neutral axis. Therefore the usual elastic flexure formula can be applied. The position of the neutral axis $\bar{y}$ can be calculated using Equation (6):

$$
\bar{y}=\frac{\sum_{i=1}^{\infty} y_{i} A_{i}}{\sum_{i=1}^{\infty} A_{i}}
$$

Where, $A_{i}$ is the area of an element $i$ and $y_{i}$ is the distance between the neutral axis of the element and the measuring axis. Considering the sizes of two components the vertical 
coordinate of the neutral axis is:

$$
I_{\bar{y}}=\left(I_{\bar{y}}\right)_{A 1}+\left(I_{\bar{y}}\right)_{A 2}
$$

$$
\bar{y}=\frac{b a\left(\frac{a}{2}\right)+d c\left(a+\frac{c}{2}\right)}{a b+d c}
$$

The moment of inertia of the whole section about the neutral axis $I_{\bar{y}}$ is the sum of the moment of inertial of each component about the neutral axis i.e:

Or;

$$
I_{\bar{y}}=\frac{b a^{3}}{12}+a b\left(\bar{y}-\frac{a}{2}\right)^{2}+\frac{d c^{3}}{12}+c d\left(\bar{y}-a-\frac{c}{2}\right)^{2}
$$

Replacing the value of the $\bar{y}$ from Equation 7 gives:

$$
I_{\bar{y}}=\frac{b a^{3}}{12}+a b\left[\frac{d c\left(a+\frac{c}{2}\right)+\frac{b a^{2}}{2}}{a b+d c}-\frac{a}{2}\right]^{2}+\frac{d c^{3}}{12}+c d\left[\frac{d c\left(a+\frac{c}{2}\right)+\frac{b a^{2}}{2}}{a b+d c}-a-\frac{c}{2}\right]^{2}
$$

Consider the transferred equivalent section. The T-shaped transformed section, of the steel type only (Figure 2), has the same behavior as the initial two component beam (Figure 1). The equation of the deflection of the equivalent steel type section in the case of small deflection is as follows:

$$
D=\frac{P L^{3}}{48 E_{s} I_{\bar{y}}}
$$

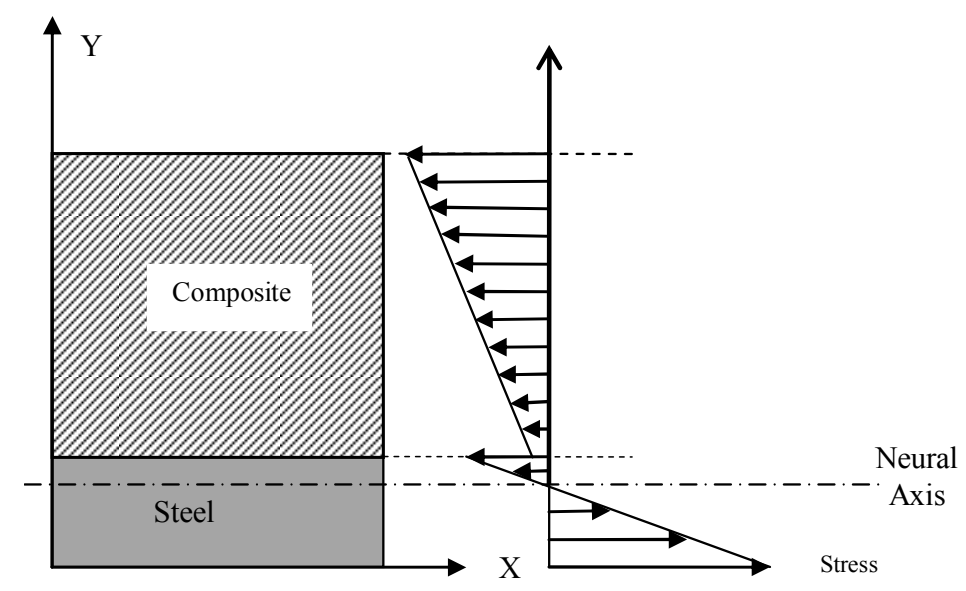

Figure 1. A schematic cross section of two component beam and tensile and compressive stress distribution diagram.

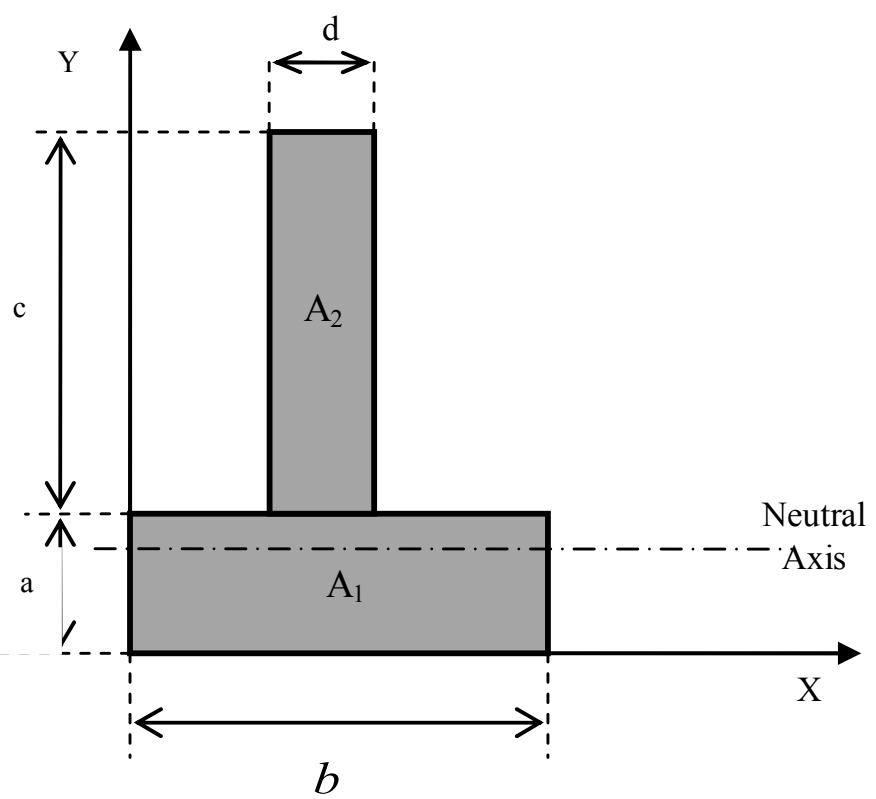

Figure 2. A schematic equivalent transferred section (spring steel only). 
According to Equation 11 the variation of the vertical load $P$ against the maximum deflection

$\mathrm{D}$ is a straight line with a gradient of $\frac{48 E_{s} I_{\dddot{y}}}{L^{3}}$. In a set of experiment, two components beam is deflected by an increasing concentrated vertical load inserted at the midpoint of the beam. The variation of $\mathrm{P}$ versus $\mathrm{D}$ is then plotted. From the slope of linear part of the curve the value of $\frac{48 E_{s} I_{\ddot{y}}}{L^{3}}$ is calculated. The length of the beam $\mathrm{L}$ and the elastic modulus of the spring steel $\left(\mathrm{E}_{\mathrm{s}}\right)$, are known, the value of $I_{\bar{y}}$ can be determined.

A numerical solution was used and a computer program was prepared to calculate the value of the modulus of the composite (E). The computer program assigns an initial value for $\mathrm{E}$ and calculates the value of the $I_{\bar{y}}$ according to Equations 5 and 10 successively. The calculated value of $I_{\bar{y}}$ is then compared with the measured value of $I_{\bar{y}}$ from the experiment. The value of $\mathrm{E}$ is increased in steps by an increment of 0.01 in each step. When the difference between the calculated and the measured values of $I_{\bar{y}}$ is near to zero, $(<0.001)$ the value of $\mathrm{E}$ is announced by the program. The computer program also calculates the value of $\bar{y}$ according to Equation 7 to compare it with the thickness of the spring steel, a, to make sure that the neutral axis intersects the spring steel region.

\section{Experimental}

\subsection{Materials and Instruments}

The above mentioned method can be applied to all types of plastic and composite sheets. To investigate the theoretical model two types of materials were used in the experiment. The characteristic of the materials are as bellow:

(a) Polypropylene sheet without reinforcement.

(b) Phenolic sheet with reinforcement part in the shape of a three dimensional fabric of polyamide yarn.

A Zwick 1446-60 tensile tester machine was adopted to perform a three point bending test. Having achieved this purpose, a steel sheet was formed to make a U-shape supports with span of $20 \mathrm{~cm}$ as shown in Figure 3- a. This steel sheet is hard enough to provide stable supports and undergoes no deformation during bending test. The U-shaped piece was then fixed, exactly in the midpoint, in the lower jaw of the tensile tester. Another flat metal bar was then fixed in the upper jaw of the tensile machine. The metal bar has rectangular cross section with the dimensions of $4 \times 30 \mathrm{~mm}$ as shown in figure $3-\mathrm{b}$. it has also a length of $50 \mathrm{~mm}$ and it is grinded to be cylindrical at the contacting edge to prevent any concentration of stress at the contacting points. Two components beam was then placed horizontally at the edges of the U-shaped supports as shown in Figure 4 and 5. The tensile tester was set to work in compression mode. The lower jaw was then moved upwards until the sharp edge of the bar contacts the midpoint of the strip. This point was adjusted to be precisely the midpoint of the beam and set to be the zero coordinates of the bending test. The lower jaw was set to move upwards with a constant speed of 20 $\mathrm{mm} / \mathrm{min}$. In this way a three point bending is performed. The vertical load applied precisely at the midpoint of the strip bends the strip which is simply supported at the ends. The value of load at the tensile tester load cell measures the vertical load. The amount of upward movement of lower jaws, recorded as extension (crush), measures the maximum deflection of the beam at the middle.

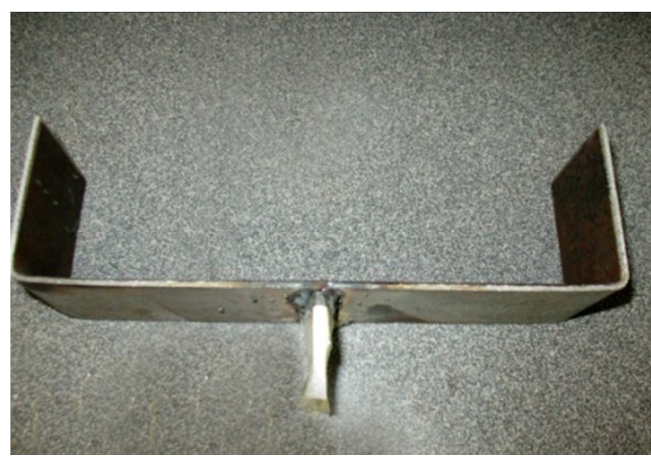

A

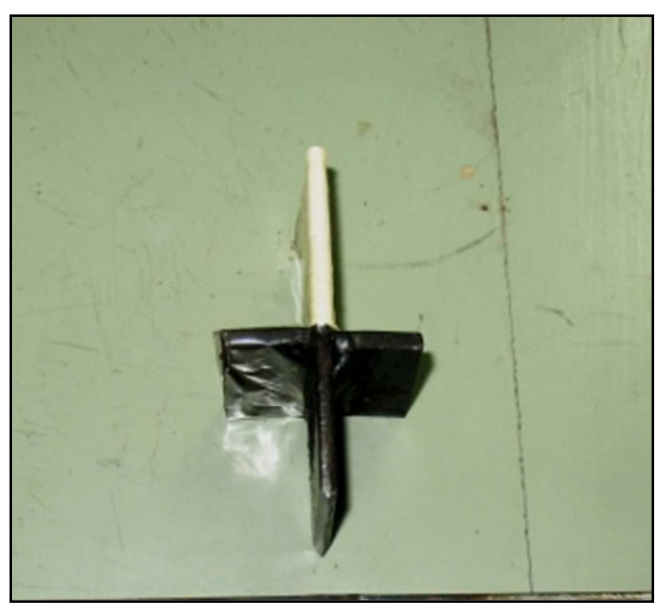

B

Figure 3. A: the U-shape supports b: the metal bar fixed in the upper jaw.

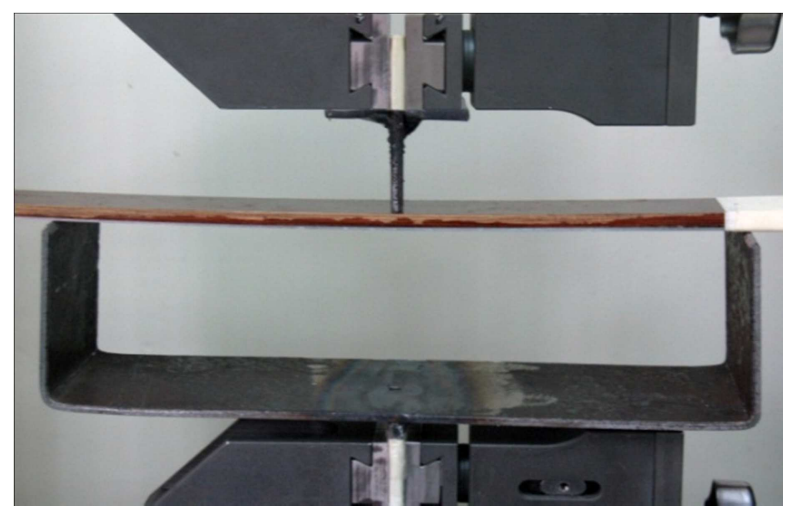

Figure 4. A tensile tester jaws adopted for flexural test. 


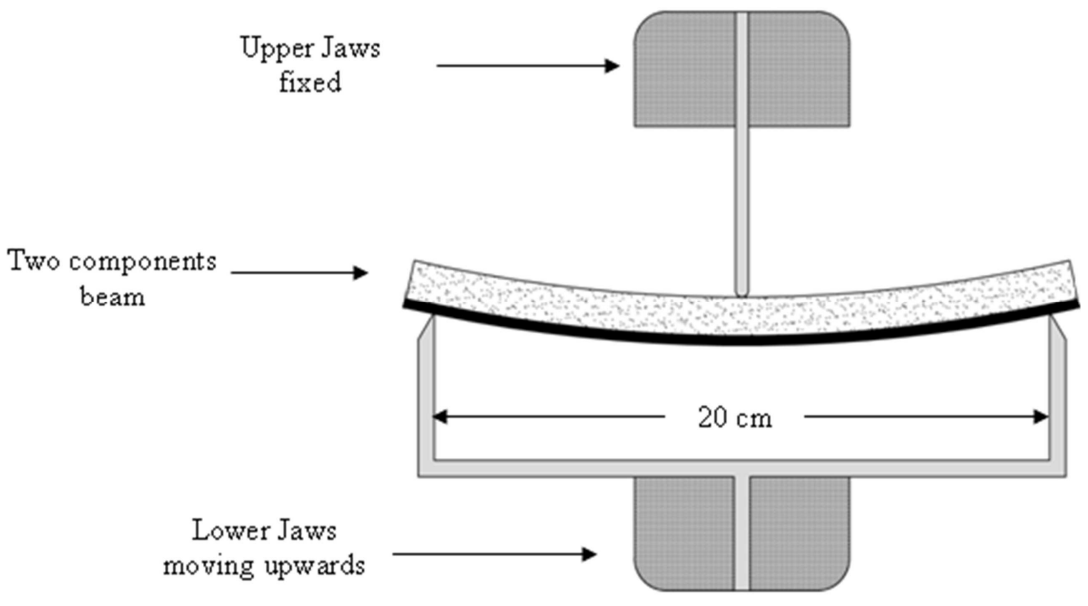

Figure 5. A schematic diagram of tensile tester jaws adopted for flexural test.

\subsection{Modulus Measurement}

\subsubsection{Steel Beam}

The spring steel strips have the length of $30 \mathrm{~cm}$, the thickness of $1 \mathrm{~mm}$ and the width of $2.54 \mathrm{~cm}$. For measuring the precise thickness of the steel sheets, a micrometer with the precision of $0.01 \mathrm{~mm}$ was used. The width of the steel strip was measured with the precision of $0.02 \mathrm{~mm}$. The spring steel strip is placed on two simple supports with a distance of $\mathrm{L}=200 \mathrm{~mm}$, the bending test was performed and the load-crush graphs was recorded.

\subsubsection{Materials modulus}

Samples of the strips were prepared with the same length and width as the spring steel strips. The longitude of each specimen must be set into defined direction. The thickness of each sample was measured by the micrometer to the nearest 0.01 of a millimeter. The average thickness of reinforced phenolic resin sheet and polypropylene sheet were 4.25 and $5.00 \mathrm{~mm}$ respectively. Each sample is then adhered on the corresponding steel sample by glue (G. Bond). The surface must completely be smeared with glue to prevent any slippage between steel and strip during the bending test. The thickness of the glue layer is very thin so it was neglected in the calculations. According to the ASTM-D790 [11], five samples must be prepared from each material sheet and the samples must be conditioned at the temperature of $23 \pm 2{ }^{\circ} \mathrm{C}$ and the relative humidity of $50 \pm 5 \%$, at least 40 hours before the experiment. According to table 93 in ASTM-D790 for three point beam, the ratio of the supports distance to the beam thickness, is equal to $60 / 1$. Thus the support distance was chosen to be $200 \mathrm{~mm}$.

The experiments were carried out at two different positions. In the first case the material strip is set to be in uppermost position thus, during bending tests, it undergoes pure compression. Bending tests were performed and the plots of the vertical load, $\mathrm{P}$, versus the maximum deflection, $\mathrm{D}$, were obtained. From the slope of the line and according to the theoretical procedure described above the value of the compressive modulus $\mathrm{E}_{\mathrm{c}}$ of material strip were determined. Similarly, in the second case bending tests were performed again with material strip in lowermost position. In this case composite undergoes pure tension and the tensile modulus of material $\mathrm{E}_{\mathrm{t}}$ can be determined.

Uniaxial tensile tests were also performed on samples. The specimens were prepared according to ASTM-D3039 [12]. Ten specimens were tested for each type of the materials.

\section{Results and Discussion}

Flexural tests were carried out on 10 different samples of spring steel and load-crush curves recorded. In the case of small deflection, according to equation (4), the variation of $\mathrm{P}$ versus D must be a straight line. Thus data points from the linear part of the curve were extracted to Excel Software and graph of variation of the vertical load $P$, versus the maximum deflection at the midpoint of the beam, D were plotted. Figure 6 shows a typical graph of P-D for spring steel. As it can be seen the data points are in a straight line with a linear regression value of $\mathrm{R}=0.98$, indicating the accuracy of small deflection equations. From the slope of lines the average elastic modulus of the spring steel, $\mathrm{E}_{\mathrm{s}}$, was calculated and is presented in Table 1.

Two-component beams were also tested in three point bending. In order to use the small deflection equation the linear region of the load-crush curves were considered and plots of the load $\mathrm{P}$ versus deflection at the midpoint $\mathrm{D}$ were plotted. Typical plots of P-D of two materials in uppermost and innermost cases are shown in Figures 7 and 8. As it can be seen clearly from the graphs the behavior of the materials is different in tension in comparison to compressive mode. From the slope of the lines and using the theory described above the average values of tensile modulus and compressive modulus of the materials were calculated and presented in Table 1. The ratios, $\mathrm{k}$, of compressive modulus to tensile modulus, for each type of the materials, were also calculated. These are shown in Table 1. This is an important factor as it can be used to define a measure of anisotropy in different types of composites and materials. The value of $\mathrm{k}$ is lower in the case of fabric reinforced phenolic resin. This can be due to the fact that the fabric part buckles more easily in 
compressive mode leading to less compressive modulus.

In order to verify the results, the values of the uniaxial tensile tests performed on the samples and the average values of the tensile moduli calculated, are presented in Table 1. The results show that the values of the tensile modules from the bending test are reasonably close to the calculated values of the tensile modules from the bending tests for both materials. Considering the values obtained from the uniaxial tests in comparison to the values obtained from this procedure, it can be concluded that, for both materials, the maximum difference is in a range of less than $10 \%$. However, apart from the experiential errors involved in the experiments, the nature of the performance of the materials in the tensile and the bending mode is different leading to difference between the measured values of the tensile modules.

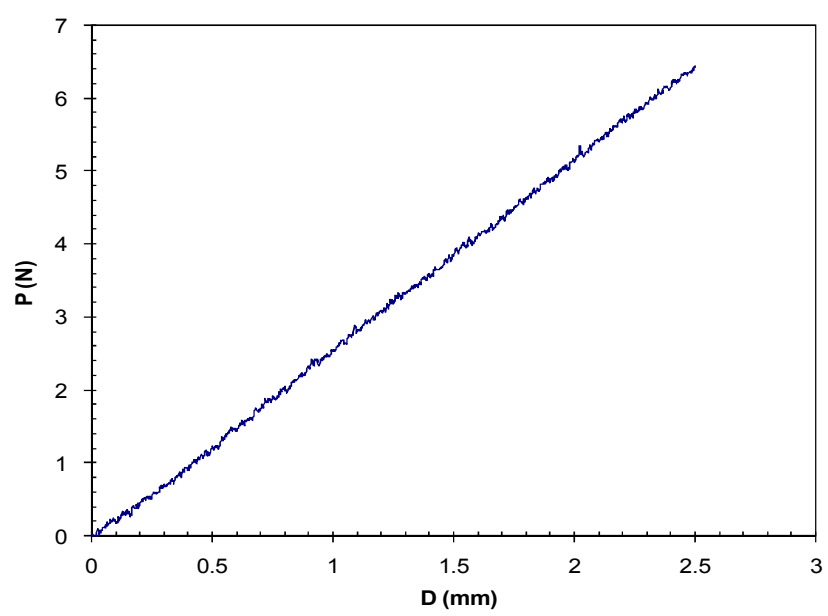

(Spring steel)

Figure 6. Plots of load $(P)$ versus maximum deflection (D).

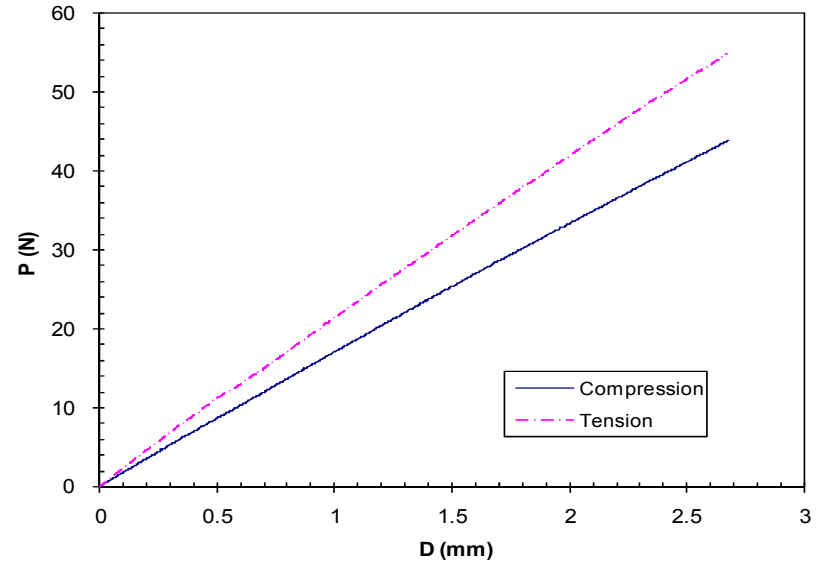

(Composite with phenolic resin + spring steel)

Figure 7. Plots of load (P) versus maximum deflection (D).

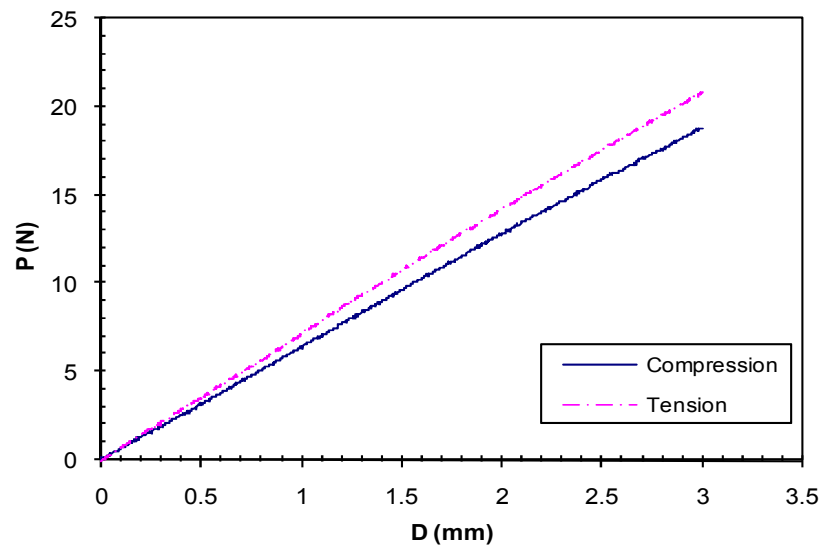

(Polypropylene strip + spring steel)

Figure 8. Plots of load $(P)$ versus maximum deflection (D).

Table 1. Average value of tensile and compressive moduli.

\begin{tabular}{|c|c|c|c|c|}
\hline \multirow[b]{2}{*}{ Material } & \multicolumn{3}{|l|}{ Bending Test } & \multirow{2}{*}{$\begin{array}{l}\text { Tensile Test } \\
\text { Tensile elastic } \\
\text { modulus (GPa) }\end{array}$} \\
\hline & $\begin{array}{l}\text { Tensile elastic modulus } \\
\mathbf{E}_{t},(\mathrm{GPa})\end{array}$ & $\begin{array}{l}\text { Compressive elastic } \\
\text { modulus } E_{c},(G P a)\end{array}$ & $\begin{array}{l}\text { Ratio of Compressive to } \\
\text { Tensile moduli (k) }\end{array}$ & \\
\hline Spring Steel & 204.50 & 204.50 & 1 & \\
\hline Reinforced Phenolic resin Composite sheet & 3.397 & 2.790 & 0.821 & 3.069 \\
\hline Polypropylene sheet & 0.812 & 0.737 & 0.908 & 0.784 \\
\hline
\end{tabular}

\section{Conclusions}

In this work a method was presented to use bending experiment to create pure tension or pure compression in some materials such as composite or plastic sheets. A two components beam deflected in a three points cantilever was used to determine tensile and compressive moduli. A tensile tester was adopted to perform the flexural tests.

Theory of equivalent section was adopted to transfer two component sections to an ordinary one component section. Using small deflection equations and theoretical analysis, values of tensile and compressive moduli of two kinds of materials were calculated. This method can be applied for all kinds of plastic and composite sheets. However, to verify the method two types of materials were used.
The results showed that the small deflection equations can be used to calculate tensile and compressive moduli with an acceptable accuracy. The results also revealed that two materials behave differently in tensile and compressive modes. For both materials, $E_{t}$ is greater than $E_{c}$. Polypropylene sheet showed lower difference between tensile and compressive moduli in comparison to the phenolic composite sheet. Higher reduction in compressive modulus in the case of fabric reinforce phenolic resin could be due to buckling of the fabric part.

\section{Acknowledgements}

The authors would like to express their sincere thanks and gratitude to the deputy of research of Isfahan University of Technology for the financial support. 


\section{References}

[1] Ting, T. C. T., S. (2006), On anisotropic elastic materials for which young modulus $\mathrm{E}(\mathrm{n})$ is independent of $\mathrm{n}$ or the shear modulus $\mathrm{G}(\mathrm{n}, \mathrm{m})$ is independent of $\mathrm{n}$ and $\mathrm{m}$, Journal of Elasticity, 81, 271-292.

[2] Cheng, X., Xiong, J. J. (2009), A novel analytical model for predicting the compression modulus of 2D PWF composites, Composite Structures, 88 (2), 296-303.

[3] Meng, M., Le, H. R., Rizvi, M. J., Grove, S. M., (2015), The effects of unequal compressive/tensile moduli of composites, $126,207-215$

[4] Sezgin, F. E., Tanoğlu, M., Eğilmez, O. Ö., Dönmez, C. (2010), Mechanical behavior of polypropylene-based honeycomb-core composite sandwich structures, Journal of Reinforced Plastics and Composites 29 (10), 1569-1579.

[5] Mujika F., Cabahjal N., Arrese A., Mondaragon I. (2006), Determination of tensile and compressive moduli by flexural tests, Polymer Testing, 25, 766-771.

[6] Reyes, G., Rangaraj, S. (2011), Fracture properties of high performance carbon foam sandwich structures, Composites Part A: Applied Science and Manufacturing 42 (1), 1-7.

[7] Jeon, J. H., Choi, D. H., Lee, P. S., Lee, K. H., Park, H. C., Hwang, W. (2006), Measuring the tensile and bending properties of nanohoneycomb structures, Mechanics of Composite Materials 42 (2), 173-186.

[8] Dobb, M. G., Ghane, M. (1996), Tensile and compressive moduli of fibres using a two-component beam system, Journal of Materials Science, 31 (17), 4445-4450.

[9] Timoshenko, S., Young, D. H. (1969), "Elements of Strength of Materials", Van Nostrand, New York, $5^{\text {th }}$ Edition.

[10] W. A. Nash, (1998), "Theory and problems of strength of materials", fourth Edition, Schaum's outline Series, McGrawHill.

[11] The American Society for Testing and Materials. (1992), "Standard test methods for flexural properties of unreinforced and reinforced plastics and electrical insulating materials", Annual books of Standards, ASTM D-790-92, 155-164.

[12] The American Society for Testing and Materials. (2000), "Standard test method for tensile properties of polymer matrix composite materials", Annual books of Standards ASTM D3039-00, Volume 15.03. 